\title{
Magnetic Localization System for Short-Range Positioning: A Ready-to-Use Design Tool
}

\author{
Luigi Ferrigno $^{(}$, Senior Member, IEEE, Marco Laracca ${ }^{(}$, Member, IEEE, Filippo Milano ${ }^{(}$, \\ Gianni Cerro $^{\circledR}$, Member, IEEE, Paolo Bellitti ${ }^{\circledR}$, Member, IEEE, Mauro Serpelloni ${ }^{\circledR}$, Senior Member, IEEE, \\ and Oscar Casas Piedrafita ${ }^{\circledR}$, Member, IEEE
}

\begin{abstract}
Magnetic localization is used in many indoor positioning applications, such as industrial, medical, and IoT, for its benefits related to the absence of line of sight needs, multipath and fading, the low cost of transmitters and receivers, and the simple development of setups made of coils and magnetic sensors. In short-range applications, this technology could bring some advantages with respect to ultrasound, laser, or RF ones. Nevertheless, fixed both the desired accuracy and the energy constraints, the optimal design of a localization system based on magnetic measurement depends on several factors: the dimension, the number and the optimal positions of the anchors, the uncertainties due to the sensing elements, and the data acquisition systems (DAQs). To preliminary fix all these parameters, suitable simulation environments allow developers to save time and money in developing localization applications. Many magnetic field simulators are available, but it is rare to find those that, considering the uncertainty due to the receiver and DAQs, are able to provide optimal anchors scenario given a target accuracy. To address this problem, this article presents a simulation tool providing the user with design requirements for given target accuracy. The aim of this article is to perform the first steps in providing a ready-to-use specification framework that given the localization domain, the mobile sensors, the DAQ characteristics, and the target accuracy and helps the developer of indoor magnetic positioning systems. The actual validity of the simulation model has been tested on a real setup.
\end{abstract}

Index Terms-Indoor positioning, magnetic localization system, magnetic sensors, tracking system, tunneling magnetoresistance (TMR), uncertainty.

\section{INTRODUCTION}

I NDUSTRIAL [1], [2] and medical applications [3] are often characterized by the need to use accurate and flexible short-range localization systems, especially when distributed and IoT-based applications are considered [4]. While the global positioning system (GPS) is the most adopted tool to localize in outdoor and long-range systems, often, it is not available in indoor scenarios, and its accuracy is poor for specific applications. Therefore, a considerable research effort is dedicated to the indoor positioning topic [5], [6]. As an example, several methods are based on wireless technologies, such as Bluetooth [7], ZigBee [8], and ultrawideband [9]. Such technologies suffer several shortcomings, such as multipath propagation, fading, uncertainty due to the antennas, uncertainty due to mobile elements in the environment, limited coverage radius, and a generally heavy signal processing phase. Some of these problems can be solved by using magnetic-based localization solutions [10], [11]. This kind of localization belongs to the anchor-based methods. Typically, the magnetic field generated by anchors in fixed and known positions is sensed by mobile elements that try to estimate their relative positions. Many solutions present in the literature optimize some aspects of this kind of localization problems. As for the energy constraints, some articles optimize the transmission power, using resonance conditions or adopting alternating and direct current [12], [13]. Some of the authors, within the framework of a relevant interest national project (PRIN), Six DoF scalable finger tracking system [14], developed a magnetic short-range localization system able to track position and orientation of an object inside a 30-cm localization domain to be used both for industrial and biomedical applications [15], [16]. In localization applications where the saving of energy on mobile nodes is important, the mobile elements could be realized by magnetic sensors. Technologies such as the Hall effect, giant magnetoresistance (GMR), tunneling magnetoresistance (TMR), and so on could be adopted for the magnetic sensing [17]-[19]. This type of localization system is very promising because, given the small size of the sensors and the very low energy consumption, it opens the doors to applications based on wearable sensors, smart gloves, and real-time monitoring of people's movements for both diagnostic and rehabilitation purposes. For these reasons, given the declared application scenarios, in the following, this article will consider this kind of localization scheme. Although these localization systems are based on very simple setups, once the type of indoor application, the areas to be covered, and the target uncertainties to be achieved have been established, the development of the optimal solution 
considers numerous variables. These include the geometric characteristics of the coils (i.e., shape, size, and number of turns), the number of anchors required, the spatial arrangement of the anchors, the minimum metrological characteristics of the magnetic sensor, and the minimum metrological characteristics of the data acquisition systems (DAQs) to which the magnetic sensors are connected.

These last two items are very important, and their behavior could deeply influence the final performance of the overall system. Regarding the metrological characteristics of the magnetic sensors to be used in the localization, since each specific sensor takes into account certain characteristics in terms of repeatability, linearity, sensitivity, frequency response, and hysteresis, it is important to estimate how these parameters influence the overall performance in order to guide the choice of the optimal sensor or the optimal ranges of use of a given sensor. The same considerations can be made with reference to the DAQ. In fact, considering that, in most applications, they are based on low-cost microcontroller architectures, it is important to estimate how the uncertainties of the acquisition system influence the accuracy of the positioning system. Given the complexity of carrying out these optimizations by following a path entirely based on experimental activities, the use of simulation systems could reduce the costs and times associated with the development of these localization systems. In the literature, there are many tools for dimensioning magnetic systems that allow defining the optimal geometries of a coil or the current amplitude necessary to have a given intensity of magnetic field in a certain region of space [20], [21]. Some companies also deal with this problem, as shown in [22]. In addition, indoor localization simulators that allow defining localization scenarios and performance are also available. Despite the existence of the mentioned systems, the authors are not aware of tools capable of evaluating the overall performance of the localization system by evaluating both the uncertainties introduced by the magnetic sensors and the uncertainties introduced by the DAQ. This work tries to contribute to this problem by proposing the development and validation of a simulation tool that, taking as input the target performance and the constraints, is able to provide the best configuration parameter set in terms of number and positions of the anchors and the number of the anchor planes, also considering the uncertainty related to the uncertainty contributions derived by an experimental characterization of the magnetic sensors and the DAQs. This article offers the possibility for researchers to have a strong reliable tool to design, implement, and test their localization systems also considering nonidealities deriving from the adoption of real devices in the experimental setup. This article is organized as follows. In Section II, a brief theoretical background is provided. In Section III, the description of the designed simulation environment is reported. In Section IV, specifications about the setup realized in [19] are described; an estimation of its nonidealities and a comparison with the optimization tool performance are provided. In Section V, the obtained results in simulations are discussed. Finally, conclusions follow in Section VI.

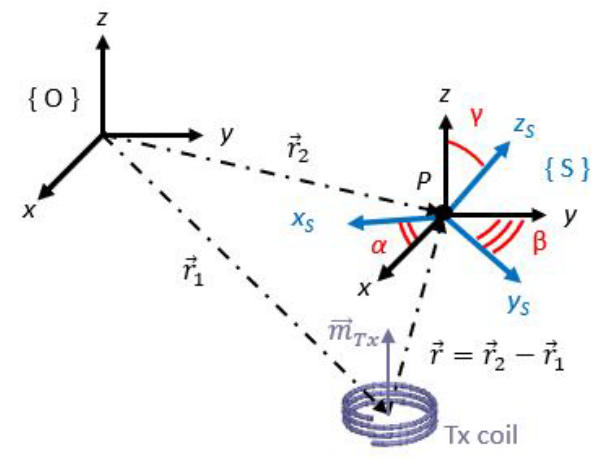

Fig. 1. Physical principle of magnetic transmission and reception using triaxial sensor.

\section{Magnetic Localization Theoretical FRAMEWORK}

The localization system considered in this article is based on the generation and measurement of alternating magnetic fields, using coils as sources ( $\mathrm{Tx}$, anchors) and magnetic field sensors as receivers ( $\mathrm{Rx}$, mobile node). In detail, the mobile node consists of a triaxial magnetic sensor, obtained by combining three orthogonal monoaxial sensors. Such a device is able to measure the intensities of the magnetic fields generated by $\mathrm{Tx}$ circular coils, placed in fixed and known positions. To improve the magnetic field intensity under the same power consumption conditions, the Tx coils have been transformed into resonant circuits by connecting appropriate capacitors in parallel to obtain resonances at different frequencies. To allow the sensor to distinguish magnetic fields deriving from different Tx coils, the transmission stage is performed in frequency division mode. This means that each Tx circuit is associated with a specific resonance frequency, different from all the others, and consequently, the Rx sensor measures a multifrequency signal where each tone is associated with a specific Tx. The measurement model is based on the magnetic dipole moment, and this provides the magnetic field generated at a point $\mathrm{P} \equiv\left(x_{P}, y_{P}, z_{P}\right)^{T}$ by a Tx coil, with angular frequency $\omega$, which is described by

$$
\vec{B}(P, t)=\frac{\mu_{0}}{4 \pi}\left(\frac{3(\vec{m} \cdot \vec{r}) \vec{r}}{\|\vec{r}\|^{5}}-\frac{\vec{m}}{\|\vec{r}\|^{3}}\right) e^{-j \omega t}
$$

where $\mu_{0}$ is the magnetic permeability of the vacuum, $\vec{m}$ is the magnetic dipole moment vector associated with the Tx coil, and $\vec{r}$ is the distance vector between the positions of Tx and point P. In (1), the distance $\|\vec{r}\|$ is small compared with the wavelength (quasi-static approximation) [23]. The triaxial sensor located at point $\mathrm{P}$ is defined by a 6-D variable $\vartheta=$ $\left(x_{p}, y_{p}, z_{p}, \alpha, \beta, \gamma\right)^{T}$. In particular, $\left(x_{p}, y_{p}, z_{p}\right)^{T}$ represents the coordinates of the point where the triaxial sensor is placed, while $(\alpha, \beta, \gamma)^{T}$ represents the orientations of the sensor axes with respect to the global frame.

Fig. 1 shows a situation of general interest in which there is a Tx coil located in point $\vec{r}_{1}$, and the triaxial sensor is located in point $\vec{r}_{2}$. The distance that separates the coil from 
the triaxial sensor is $\|\vec{r}\|=\left\|\overrightarrow{r_{2}}-\overrightarrow{r_{1}}\right\|$, while the inertial triad of the sensor is rotated, respectively, $(\alpha, \beta, \gamma)$ angles with respect to the $(x, y, z)$ axes. In this context, it is possible to determine the theoretical field measured by the triaxial sensor by means of the following equation:

$$
\vec{B}_{S}(P, t)=\sum_{i=1}^{N_{T x}} R_{\alpha} R_{\beta} R_{\gamma} \vec{B}_{i}(P, t)
$$

where $\vec{B}_{i}(P, t)$ is the field generated by the Tx coil on the basis of (1), while $R_{\alpha}, R_{\beta}$, and $R_{\gamma}$ are rotation matrices related, respectively, to $x$-, $y$-, and $z$-axes. Once the signals have been acquired, a fast Fourier transform (FFT) operation is carried out to get the root-mean-square (rms) values of all the frequency components of the magnetic field generated by the Tx coils in the point P. Finally, the position and orientation of the mobile node can be estimated using a numerical technique. In particular, the estimate is made by minimizing a cost function (3) with respect to the variable $\vartheta$ through the Nelder-Mead algorithm [24]. In (3), $\tilde{\vec{B}}_{\mathrm{rms}, i}$ represents the measured magnetic field vector corresponding to the $i_{t h}$ Tx circuit, while $\vec{B}_{\mathrm{rms}, i}$ is the theoretical magnetic field, that is, the one that should be measured whenever the position and orientation of the sensor were equal to $\vartheta$, according to (2)

$$
F(\vartheta)=\sum_{i=1}^{N_{T x}}\left\|\tilde{\vec{B}}_{\mathrm{rms}, i}-\vec{B}_{\mathrm{rms}, i}(\vartheta)\right\| .
$$

\section{Designed Optimization Tool}

Fig. 2 shows a conceptual scheme of the developed optimization tool. It has been developed in a MATLAB environment, and it has been parameterized on the following input quantities:

- localization domain size;

- maximum number of active planes in the localization domain;

- maximum number of Tx coils per each active plane;

- contribution of accuracy due to the magnetic sensors;

- contribution of accuracy due to the DAQ;

- allowed accuracy for the positioning required by the application (target performance).

All input contributions are used by the simulator to compute localization on a predefined set of uniformly distributed points within the localization domain, and according to the obtained positioning errors (i.e., the difference between the estimated and the real pose, as detailed in the following), it outputs the best configuration parameter set to be able to achieve the target performance (positioning errorless or equal to the maximum admitted error) under real component nonidealities and geometric/electrical features' constraints. In particular, the best configuration parameter set is identified by the optimal number of active domain planes and Tx coils.

Getting into details, Fig. 3 describes the content of the black box. It is an iterative procedure where, starting from the minimum configurations in terms of coils and active planes, it computes the localization on a predefined points' cloud. After that, it computes the localization errors, and whenever

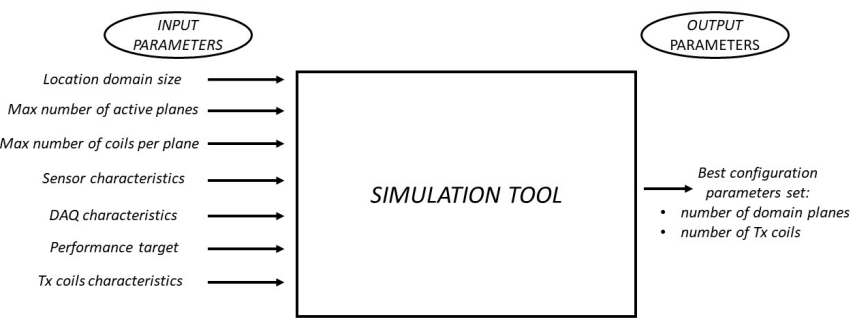

Fig. 2. Block diagram of the developed simulation tool.

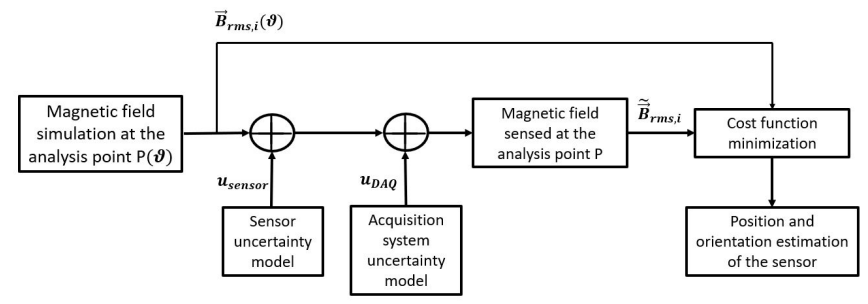

Fig. 3. Flowchart of the operations inside the simulator.

the maximum obtained error is below the error threshold given as input, it stops computing and returns such configuration as optimal. Conversely, if the maximum error overcomes the threshold, it continues iterating by adding anchor coils and active planes to the simulation scenario. Such a procedure comes to an end in a finite time since it is upper bounded by the inputs regarding the maximum number of coils and planes that are affordable by the designer.

In a generic iteration, the localization procedure works as follows. The first performed operation is to calculate, by means (1), the magnetic field $\left(\vec{B}_{\mathrm{rms}, i}\right)$ at point $\mathrm{P}$ (one in the cloud) (see Fig. 1) characterized by the 6 -D variable $\theta$. Then, the uncertainty models of both the sensor and the acquisition system are considered. That is, the computed magnetic field deviates from its correct value by adding uncertainty contributions. Such value is then transformed according to (2) and named $\widetilde{\vec{B}}_{\mathrm{rms}, i}$. The latter is considered as the magnetic field sensed by the triaxial magnetic sensor. Finally, by minimizing (3), it is possible to estimate the point $\tilde{P}$ that characterizes the position and orientation of the triaxial sensor (the mobile node). Such a procedure is repeated for each point in the points' cloud.

According to (4), the positioning error $(\|\epsilon\|)$ is defined as the distance between the true sensor position and the estimated one. In (5), $\epsilon_{i}$ represents the committed errors on the $i$ th coordinate (e.g., $x, y$, and $z$ ), calculated as the difference between the generic true coordinate $\left(\theta_{i}\right)$ and the generic estimated coordinate $\left(\tilde{\theta}_{i}\right)$

$$
\begin{aligned}
\|\epsilon\| & =\sqrt{\epsilon_{x}^{2}+\epsilon_{y}^{2}+\epsilon_{z}^{2}} \\
\epsilon_{i} & =\theta_{i}-\tilde{\theta}_{i}, \quad i \in\{x, y, z\} .
\end{aligned}
$$

The coordinate $\tilde{\theta}_{i}$ can be estimated through experimental data obtained by a measurement campaign (in this case, $\|\epsilon\|$ is called "experimental positioning error"). On the other hand, if the coordinate $\tilde{\theta}_{i}$ is estimated through simulated data (obtained by means of the optimization tool), $\|\epsilon\|$ is called 


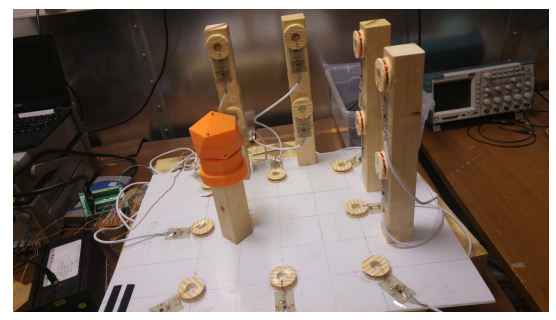

(a) The realized experimental set-up.

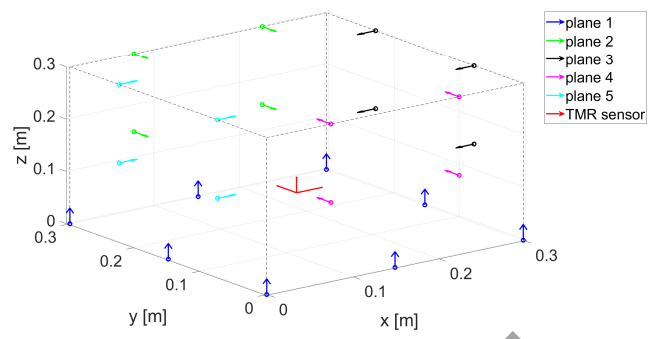

(b) Schematic diagram of the realized set-up.

Fig. 4. Realized setup. (a) Realized experimental setup. (b) Schematic of the realized setup.

"simulated positioning error." In particular, in this last case, the estimation of $\tilde{\theta}_{i}$ is made starting from the simulated magnetic field $\tilde{\vec{B}}_{\mathrm{rms}, i}$ sensed by the triaxial magnetic sensor, considering the uncertainty contributions of the measurement system.

\section{EXPERIMENTAL VALIDATION}

\section{A. Realized Setup}

The performance of the proposed optimization tool has been preliminarily evaluated with respect to an experimental setup. In detail, the setup proposed in [19] has been adopted. This setup is intended to solve short-range localization related to medical problems in which smart gloves can retrieve the position of fingers to evaluate the performance of some hand gesture movements. In these kinds of problems, requirements such as the weight and the power consumption of the positioning system mobile nodes of the positioning systems are a very important issue, and the solution based on miniaturized and low-power magnetic sensors could be preferable with respect to mobile nodes based on coils. As shown in Fig. 4(a), the considered experimental setup is composed of 24 anchors (i.e., Tx coils at a fixed frequency), placed on five planes. In the bottom plane, there are eight coils, and all the lateral planes contain four coils per plane. For the sake of clarity, some of the vertical coils are removed to take the photograph to clearly show the inner part of the setup. Fig. 4(b) contains a schematic of the anchor coils placement in which different colors represent coils placed on different planes. The overall localization domain is a cube with a length of $30 \mathrm{~cm}$. Each one of the Tx coils has a circular shape with an internal radius of $10 \mathrm{~mm}$ and a height of $6 \mathrm{~mm}$. Each coil is made using a wire with a diameter equal to $0.3 \mathrm{~mm}$ that realizes 15 turns. To optimize transmission efficiency, each anchor coil is connected in parallel to a capacitor in order to obtain resonance condition at the desired frequency of that Tx coil.

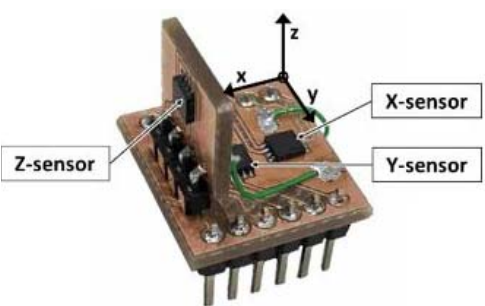

Fig. 5. Snapshot of the adopted triaxial TMR sensor.

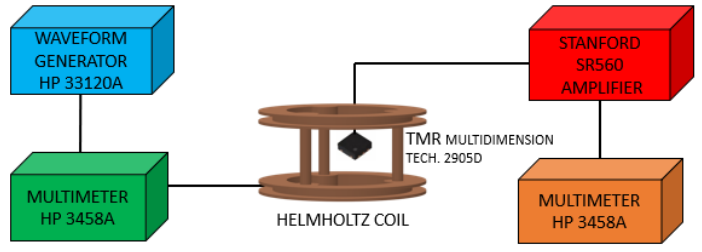

Fig. 6. Schematic of the developed setup for the TMR characterization.

All the considered coils are supplied by a sinusoidal alternating current with an amplitude of about 50-mA rms per coil and frequencies ranging from 26 to $40 \mathrm{kHz}$. Looking at Fig. 4(a), the mobile node is closed in the orange fixture that allows it to be rotating with known and fixed degrees. As for the sensor that composes the mobile node, it is a triaxial sensor made using three TMR magnetic sensors (namely, the 2905D manufactured by MultiDimension Technology).

Fig. 5 shows the developed board for the arrangement of the TMR sensors. In this photograph, the placement of each one of the three monoaxial sensors on the triaxial support can be highlighted. The output signals of the three TMR sensors are conditioned by three AD620 instrumentation amplifiers, with a gain of 102.56 , and they are acquired by means of a TiePie Handiscope HS5 DAQ.

\section{B. Estimation of Nonidealities Related to the Experimental Setup}

The nonidealities related to the mobile node of the considered experimental setup to be considered in the developed optimization tool have been retrieved, as detailed in the following.

1) As for the DAQ, the uncertainty contribution has been estimated starting from metrological characteristics exported in the manufacturer manual [25]. In particular, for the considered measurement range case, accuracy is equal to $0.25 \%$ Considering the worst case accuracy and a rectangular distribution of the accuracy, the uncertainty contribution due to the data acquisition range can be estimated.

2) As for the TMR sensors, since the metrological characteristics furnished by the manufacturer are not exhaustive, a preliminary experimental characterization has been carried out to assess the different uncertainty contributions.

To characterize the TMR, an experimental setup was arranged. The block diagram is shown in Fig. 6. 


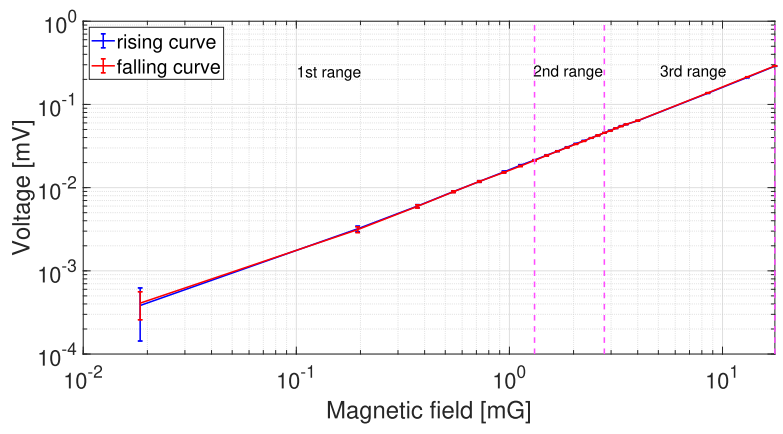

Fig. 7. TMR characterization curve divided into the three explored ranges (first range: $18.5 \mu \mathrm{G}-1.31 \mathrm{mG}$; second range: $1.31-2.78 \mathrm{mG}$; and third range: 2.78 to $17.6 \mathrm{mG})$

Looking at Fig. 6, the sensor was positioned inside a certified Helmholtz coil, in order to be subjected to a uniform field. An HP 33120A waveform generator was used to power the Helmholtz coil, and an HP 3458A multimeter measures the current flowing in the coil. The sensor output was amplified and filtered through a Stanford SR560 amplifier and acquired by a reference 8.5-digit digital multimeters, namely, the Hewlett and Packard HP 3458A. The TMR experimental characterization was carried out by considering 24 measurement points, in terms of the amplitude of the magnetic field that are equally distributed within the range from $18.5 \mu \mathrm{G}$ up to $17.6 \mathrm{mG}$ at a frequency value equal to $33 \mathrm{kHz}$ that corresponds to the half value of the considered excitation range reported in Section IV-A. As for the amplitude range, it contains the values of the magnetic field that can be experimented with, given the anchor system described in the previous section, in each point of the localization domain. As for the frequency selected for the characterization, a preliminary stability analysis made in the whole frequency range has highlighted as sensor behavior is very stable in this range. For each point of the considered range, 20 measurements were repeated, spanning the range both in the rise way and the fall way to estimate the hysteresis contribution. The obtained experimental characterization results are shown in Fig. 7. Looking at 7 , it can be highlighted that the whole characteristic can be divided into three zones with different sensitivity and repeatability; the characteristics of these three zones have been considered to estimate the uncertainty contribution. In addition, to warrant a sufficient number of measurement points for each zone, each of them was explored by using eight points. The estimated uncertainty contribution, for each zone, was obtained the repeatability $\left(u_{\mathrm{REP}}\right)$, the sensitivity $\left(u_{\mathrm{SEN}}\right)$, the hysteresis $\left(u_{\mathrm{HYS}}\right)$, and the nonlinearity $\left(u_{\mathrm{N} . \mathrm{L} .}\right)$. In the three considered ranges, the obtained values for $u_{\mathrm{TMR}}$ are equal to $[1.44,0.91,1.65] \mathrm{mV} / \mathrm{G}$ with sensitivity values equal to $[16.3,16.6,15.8] \mathrm{mV} / \mathrm{G}$

$$
u_{\mathrm{TMR}}=\sqrt{u_{\mathrm{REP}}^{2}+u_{\mathrm{SEN}}^{2}+u_{\mathrm{HYS}}^{2}+u_{\mathrm{N} . \mathrm{L}}^{2}} .
$$

The division of the TMR characteristic was carried out with the aim to reduce the nonlinearity error of the sensor. In particular, the choice has been made looking for a tradeoff between the complexity of the obtained sensor characteristic and the reduction of the nonlinearity effect of the sensor. Considering
TABLE I

UnCertainty Contributions Due to Repeatability, Sensitivity, HYSTERESIS, AND NONLINEARITY IN THE THREE EXPLORED RANGES

\begin{tabular}{cccc}
\hline \hline Parameters $[\mathrm{mV} / \mathrm{G}]$ & $1^{\text {st }}$ range & $2^{\text {nd }}$ range & $3^{\text {rd }}$ range \\
\hline $\mathrm{u}_{R E P}$ & 1.38 & 0.03 & 0.024 \\
$\mathrm{u}_{S E N}$ & 0.34 & 0.88 & 1.64 \\
$\mathrm{u}_{H Y S}$ & 0.19 & 0.18 & 0.11 \\
$\mathrm{u}_{N . L .}$ & 0.037 & 0.055 & 0.083 \\
\hline \hline
\end{tabular}

TABLE II

COORDINATES OF THE EXAMINED POINTS

\begin{tabular}{|c|c|c|c|}
\hline Positions & $\bar{x}[\mathbf{m m}]$ & $\bar{y}[\mathrm{~mm}]$ & $\mathrm{z}[\mathrm{mm}]$ \\
\hline 1 & 75 & 225 & 20 \\
\hline 2 & 150 & 75 & 20 \\
\hline 3 & 150 & 150 & 170 \\
\hline 4 & 75 & 150 & 170 \\
\hline 5 & 150 & 225 & 20 \\
\hline 6 & 225 & 225 & 20 \\
\hline
\end{tabular}

the whole characteristics, the uncertainty contribution due to the nonlinearity was equal to $0.34 \mathrm{mV} / \mathrm{G}$ while considering the divided characteristic that we have obtained the values reported in Table I that also summarizes the other obtained experimental values of the considered uncertainty contributions in the three considered ranges.

\section{Validation of the Optimization Tool}

To validate the developed optimization tool, we compare results obtained in the simulated environment with those deriving from experimental measurements. Using the experimental setup described in Section IV-A, the simulation tool validation was made on six experimental positions within the considered localization domain. Table II shows the Cartesian coordinates of test points: for all cases, the orientation angles are set to 0 . This choice was made to grant a more accurate arrangement of the test setup. In fact, by setting the orientation angles to zero, it is easier to accurately place the three-axial TMR sensor inside the used support, rather than using three generic rotation angles $(\alpha, \beta$, and $\gamma)$. This choice does not affect the generality of the presented experimental validation. In fact, depending on the number of planes and the number of active coils, there is always one of three monoaxial sensors working better than others.

The experimental setup and the simulation tool were tested using the same operating conditions (i.e., number of Tx coils equal to 24 , number of domain planes equal to 5 , number of iterations equal to 10, the same arrangement of sensors, and so on). In the simulator, the uncertainty contribution, due to the magnetic sensor (and experimentally obtained by the TMR sensor characterization), was added to the uncertainty due to the acquisition system. Fig. 8 shows a comparison of the obtained results in terms of the positioning errors $(\|\epsilon\|)$ obtained from the experimental and simulated data. Fig. 8 shows the good agreement, estimated with a confidence level equal to $95 \%$, between the experimental results and those obtained with the proposed optimization system in a 


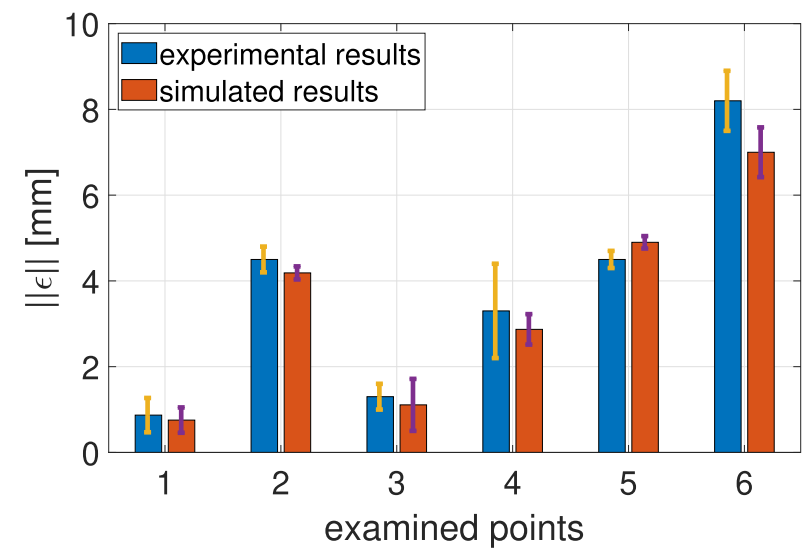

Fig. 8. Obtained positioning errors for the experimental data (in blue) and the simulated data (in red).

simulated environment. This confirms the goodness of the realized simulation tool.

\section{Magnetic Localization Design Using the DEVELOPED OPTIMIZATION TOOL}

Once the reliability of the proposed optimization tool in emulated environment was verified, it has been used to simulate a number of scenarios that starting from the considered application could help the developer to find optimal configurations given a target accuracy and to evaluate the effect of changes of the magnetic sensors, the effect of changes in the $\mathrm{DAQ}$, and the effect of changes in the number of coils or changes of the coil positioning. In addition, the tool can be also used to estimate if some zones of the localization domain warrant better or worsen accuracy with respect to other zones. In this way, in some applications, limitations in the localization domain can be preventively estimated.

\section{A. Variability Range of the Considered Parameters}

Since there are a great number of the input parameters of the simulation tool, some of these were considered fixed (the less significant ones), and the others were considered variable inside certain ranges arise by the specific application field (the chosen values are mainly focused on the application related to the project [14]). In the following, details about the considered values for each considered input parameter are reported.

1) The considered localization domain was a cube with a fixed side of $50 \mathrm{~cm}$.

2) Since the localization domain is a cube, the number of domain planes was selected inside a range from 1 to 6 .

3) The number of Tx coils per plane was selected inside a range from 4 to 9 .

4) Considering the typical value of the commercial measurement systems, their accuracy contribution Acc\% (both DAQ and triaxial sensing system) was selected inside a range from $0.1 \%$ to $0.3 \%$.

5) The maximum admitted positioning error was ranged from 0.1 to $10 \mathrm{~mm}$ according to the main indoor magnetic localization application.

6) In order to focus the design analysis on the parameters $2-5$, the geometric and electrical characteristics of the

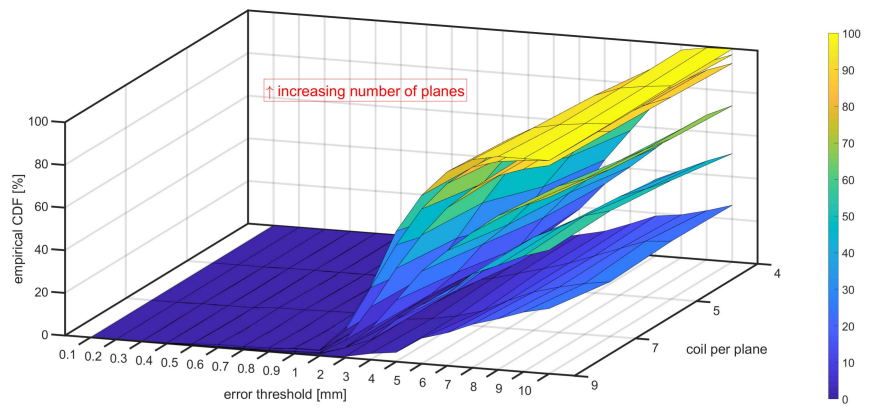

Fig. 9. Empirical CDF of the positioning error by varying the number of Tx coils per planes and the number of planes with Acc $_{\%}=0.3 \%$.

Tx coils were considered fixed and equal to ones used for the experimental validation reported in Section IV-A.

Finally, the magnetic localization design was made on a total of 343 points equally spaced within the chosen localization domain. Regarding the orientations of these points, expressed through the angular variables $\alpha, \beta$, and $\gamma$, they have been chosen randomly and uniformly distributed in $\left[0^{\circ}, 360^{\circ}\right]$.

As far as the spatial discretization of the localization domain, two considerations should be made: there is no specific rule to choose the points to be considered in the design phase since there are no better or worse positions (in fact, in a specific position, one sensor axis can work better than others and vice versa); in the designing phase of the magnetic positioning system, the spatial discretization of the considered domain (the number of the sensor positions and orientations in the localization domain) can affect the suitability of the obtained results (the greater the number of considered positions, the better the quality of the obtained design results).

For each examined point, 100 repeated measurements were made and processed to estimate the rms values of the sensed magnetic fields on the three-sensor axis.

\section{B. Obtained Results Using the Optimization Tool}

A number of simulations were made varying the considered parameters inside the specified ranges. For each simulated configuration, an empirical cumulative distribution function (CDF) was obtained, which indicates the percentage of the number of points that have a positioning error lower than predefined error thresholds. Figs. 9-11 show the obtained results where, for each CDF, the number of Tx coils per plane is shown on the $x$-axis, the $y$-axis represents the positioning error thresholds, and, finally, the $z$-axis represents the percentage of analyzed points with positioning errors below the threshold. Furthermore, these figures are parameterized according to the employed active planes (ranging from 1 to 6), and therefore, each figure contains six plots. Furthermore, the colors of the figures and, therefore, the colorbar are in accordance with the values of the CDF. Figures are presented in inverted order with respect to the accuracy $\left(\mathrm{Acc}_{\%}\right)$ of the measurement system. In detail, Fig. 9 reports the results obtained using an accuracy value equal to the one employed in a real setup. Considering such accuracy $(0.3 \%)$ and a target error equal to $8 \mathrm{~mm}$, our 


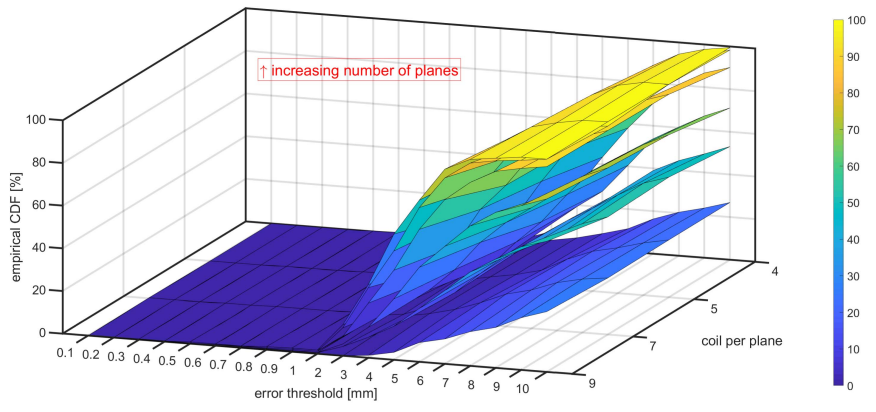

Fig. 10. Empirical CDF of the positioning error by varying the number of Tx coils per planes and the number of planes with $\mathrm{Acc}_{\%}=0.15 \%$.

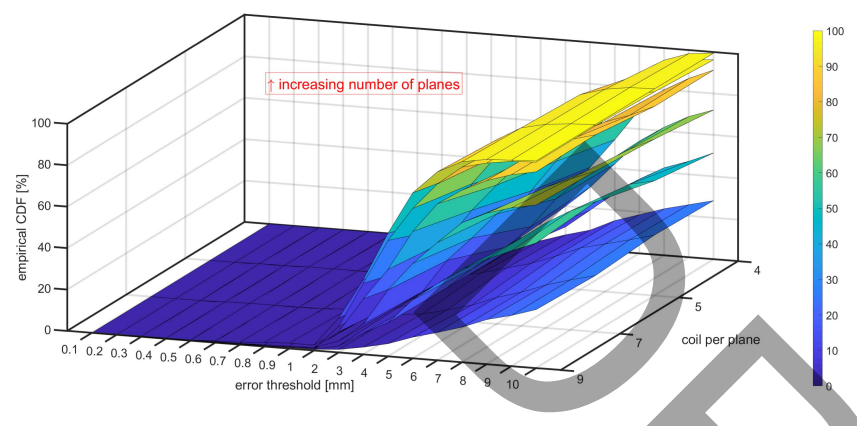

Fig. 11. Empirical CDF of the positioning error by varying the number of Tx coils per planes and the number of planes with Acc $\%=0.1 \%$

TABLE III

Comparison of All Simulated Configurations When the Error THRESHOLD IS EQUAL TO $6 \mathrm{~mm}$ WITH ACC $\%=0.30 \%$

\begin{tabular}{ccccccc}
\hline \hline & \multicolumn{7}{c}{ Domain planes } \\
\hline Tx coils per plane & 1 & 2 & 3 & 4 & 5 & 6 \\
\hline 4 & 17.6 & 35.8 & 50.4 & 72.0 & 76.9 & 88.6 \\
5 & 17.5 & 38.5 & 56.0 & 77.8 & 86.0 & 92.7 \\
7 & 18.1 & 43.7 & 61.2 & 84.5 & 90.9 & 95.0 \\
9 & 20.4 & 44.1 & 63.5 & 83.9 & 94.1 & 97.6 \\
\hline \hline
\end{tabular}

optimization tool provides as output that, with four Tx coils per planes and five active planes, the required target error is achieved $87.7 \%$ of cases. By decreasing the nonideality level of the system, i.e., by considering a lower percentage as accuracy, e.g., $0.15 \%$ (see Fig. 10, the same configuration ensures the target performance in $89.2 \%$ of cases. Finally, if a further decrease in accuracy percentage is inputted to the system [e.g., $0.1 \%$ (see Fig. 11)], the same configuration allows having $92.4 \%$ of tested points being under the target error threshold. As general remarks, there is a good behavior of the system when the error threshold is relatively high, from 10 down to $5 \mathrm{~mm}$. By reducing the error threshold, we have a reduction in the percentages. In any case, these percentages show an improvement when the number of Tx coils per plane and the number of domain planes increase. The performance worsens, however, when the considered accuracy value increases.

As a further example, a comparison using a 6-mm error target is shown in Table III, considering an accuracy value equal to $0.30 \%$. The values shown in Table III are expressed as a percentage, and they are obtained by the empirical CDF.
It can be noted that it is not possible to guarantee localization performance with positioning errors of less than $5 \mathrm{~mm}$ with a CDF of $100 \%$. In fact, considering accuracy of $0.3 \%$, we need nine Tx coils per plane and six active planes to ensure that $90 \%$ of the points have errors below $5 \mathrm{~mm}$.

\section{CONCLUSiON}

This article provides the design and optimization criteria for a magnetic localization system based on circular transmitting coils and a triaxial magnetic field sensor as a receiver. It is developed in a simulation environment to test the system with variable dimension, number, positions, shape, and current of anchors, without needing to physically implement the system, thus saving money and time resources. It is able to consider nonidealities of real systems, both in terms of acquisition (data acquisition unit accuracy) and measurement (limited sensitivity, nonlinearity, hysteresis, and repeatability of suitable magnetic sensors). The tool has been compared with a real setup and performance, considering that a confidence level equal to $95 \%$ is proven to be compatible. Finally, it is possible to state that the developed tool is able to output the optimal configuration, to be later physically implemented, under power, space, time constraints, and target performance levels to be achieved. The important statement that comes from this article is that, despite the simulation environment, if very low errors are required and limited resources, both in terms of usable devices and poor accuracy levels of the adopted sensors, are available, it is not possible to get an optimal configuration allowing $100 \%$ achievable performance. On the other hand, an important output of the presented results is that, in some cases, it is not necessary to have full coverage of the localization domain in terms of anchors to get high levels of performance. Therefore, a tradeoff between achievable performance and a limited budget for system setup is generally present. The developed tool has been designed as modular in order to allow the further introduction of novel uncertainty contributions (i.e., magnetic environmental noise) that system different from the one presented could give in their physical implementation. The future development of the optimization tool presented in this work involves the evaluation and comparison of the current implementation with other types of optimization solvers based on stochastic mathematical methods, genetic algorithms, and particle swarm optimization method [26]-[28].

\section{REFERENCES}

[1] L. Angrisani, F. Bonavolontà, G. Cavallo, A. Liccardo, and R. S. L. Moriello, "On the measurement uncertainties of $\mathrm{THz}$ imaging systems based on compressive sampling," Measurement, vol. 116, pp. 83-95, Feb. 2018.

[2] G. Cerro et al., "An accurate localization system for nondestructive testing based on magnetic measurements in quasi-planar domain," Measurement, vol. 139, pp. 467-474, Jun. 2019.

[3] L. Ferrigno et al., "A real-time tracking system for tremor and trajectory estimation in Parkinson's disease affected patients," in Proc. IEEE Int. Symp. Med. Meas. Appl. (MeMeA), Jun. 2020, pp. 1-6.

[4] F. Khelifi, A. Bradai, A. Benslimane, P. Rawat, and M. Atri, "A survey of localization systems in Internet of Things," Mobile Netw. Appl., vol. 24, no. 3, pp. 761-785, Jun. 2019. 
[5] F. Zafari, A. Gkelias, and K. K. Leung, "A survey of indoor localization systems and technologies," IEEE Commun. Surveys Tuts., vol. 21, no. 3, pp. 2568-2599, 3rd Quart., 2019.

[6] A. Yassin et al., "Recent advances in indoor localization: A survey on theoretical approaches and applications," IEEE Commun. Surveys Tuts., vol. 19, no. 2, pp. 1327-1346, 2nd Quart., 2017.

[7] O. Cruz, E. Ramos, and M. Ramirez, "3D indoor location and navigation system based on Bluetooth," in Proc. CONIELECOMP, 21st Int. Conf. Electr. Commun. Comput., Feb. 2011, pp. 271-277.

[8] J. Niu, B. Wang, L. Shu, T. Q. Duong, and Y. Chen, "ZIL: An energy-efficient indoor localization system using ZigBee radio to detect WiFi fingerprints," IEEE J. Sel. Areas Commun., vol. 33, no. 7, pp. 1431-1442, Jul. 2015 .

[9] S. Krishnan, P. Sharma, Z. Guoping, and O. H. Woon, "A UWB based localization system for indoor robot navigation," in Proc. IEEE Int. Conf. Ultra-Wideband, Sep. 2007, pp. 77-82.

[10] D. Lin and X. Chen, "Mathematical models of 3D magnetic field and 3D positioning system by magnetic field," Appl. Math. Inf. Sci., vol. 8, no. 4, p. 1647, 2014.

[11] W. Kim, J. Song, and F. C. Park, "Closed-form position and orientation estimation for a three-axis electromagnetic tracking system," IEEE Trans. Ind. Electron., vol. 65, no. 5, pp. 4331-4337, May 2018

[12] G. De Angelis et al., "An indoor AC magnetic positioning system," IEEE Trans. Instrum. Meas., vol. 64, no. 5, pp. 1267-1275, May 2015.

[13] J. Blankenbach and A. Norrdine, "Position estimation using artificial generated magnetic fields," in Proc. Int. Conf. Indoor Positioning Indoor Navigat., Sep. 2010, pp. 1-5.

[14] The 6 DOF Finger Tracking Project-A Short Range Wireless Positioning System. Accessed: Jul. 20, 2020. [Online]. Available: http://6doftracking.unipg.it./

[15] V. Pasku, A. De Angelis, M. Dionigi, G. De Angelis, A. Moschitta, and P. Carbone, "A positioning system based on low-frequency magnetic fields," IEEE Trans. Ind. Electron., vol. 63, no. 4, pp. 2457-2468, Apr. 2016.

[16] D. Capriglione, G. Cerro, L. Ferrigno, F. Milano, and A. Moschitta, "A multi-frequency approach to mitigate the performance degradation of a magnetic positioning system under CW disturbance conditions," Measurement, vol. 161, Sep. 2020, Art. no. 107842.

[17] S. Song et al., "6-D magnetic localization and orientation method for an annular magnet based on a closed-form analytical model," IEEE Trans. Magn., vol. 50, no. 9, pp. 1-11, Sep. 2014.

[18] C. Hu, M. Q.-H. Meng, and M. Mandal, "A linear algorithm for tracing magnet position and orientation by using three-axis magnetic sensors," IEEE Trans. Magn., vol. 43, no. 12, pp. 4096-4101, Dec. 2007.

[19] G. Cerro et al., "On a finite domain magnetic localization by means of TMR triaxial sensors," in Proc. IEEE Int. Instrum. Meas. Technol. Conf. (I2MTC), May 2020, pp. 1-6.

[20] C. Hu, T. Ma, and M. Q.-H. Meng, "Sensor arrangement optimization of magnetic localization and orientation system," in Proc. IEEE Int. Conf. Integr. Technol., Mar. 2007, pp. 311-315.

[21] A. Sheinker, B. Ginzburg, N. Salomonski, L. Frumkis, and B.-Z. Kaplan, "Localization in 3-D using beacons of low frequency magnetic field," IEEE Trans. Instrum. Meas., vol. 62, no. 12, pp. 3194-3201, Dec. 2013.

[22] J. Kostak. Indoor Location Tracking and Positioning. Accessed: Jul. 20, 2020. [Online]. Available: https://www.sewio.net/indoor-locationtracking-and-positioning/

[23] D. J. Griffiths, Introduction to Electrodynamics. Upper Saddle River, NJ, USA: Prentice-Hall, 2007.

[24] K. I. M. McKinnon, "Convergence of the Nelder-Mead simplex method to a nonstationary point," SIAM J. Optim., vol. 9, no. 1, pp. 148-158, Jan. 1998, doi: 10.1137/S1052623496303482.

[25] Handyscope HS5 USB Oscilloscope. Accessed: Jul. 20, 2020. [Online]. Available: https://www.tiepie.com/en/usb-oscilloscope/handyscope-hs5

[26] S. Tsatalas et al., "A novel multi-magnetometer facility for on-ground characterization of spacecraft equipment," Measurement, vol. 146, pp. 948-960, Nov. 2019.

[27] E. Carrubba, A. Junge, F. Marliani, and A. Monorchio, "Particle swarm optimization for multiple dipole modeling of space equipment," IEEE Trans. Magn., vol. 50, no. 12, pp. 1-10, Dec. 2014.

[28] S.-D.-J. Kakarakis, S. T. Spantideas, N. C. Kapsalis, C. N. Capsalis, and A. Junge, "A software-based calibration technique for characterizing the magnetic signature of EUTs in measuring facilities," IEEE Trans. Electromagn. Compat., vol. 59, no. 2, pp. 334-341, Apr. 2017. 